\title{
Velocity and turbulence affected by various vegetations in open channel
}

\author{
Zhilin Sun ${ }^{1}$, Xin Meng ${ }^{1, *}$, Yubao Zhou ${ }^{2}$ \\ ${ }^{1}$ Ocean College, Zhejiang University, Hangzhou 310058, China \\ ${ }^{2}$ Faculty of Civil Engineering and Geosciences, Delft University of Technology, Netherland
}

\begin{abstract}
Water energy is a conventional source of source that is widely available in nature. It can be easily converted into high quality secondary energy-electric energy through hydroelectric power stations. Hydropower is not only a widely used conventional energy source, but also a renewable energy source. Moreover, hydroelectric power has no pollution to the environment. Therefore, water energy is an inexhaustible and high-quality energy source among many energy sources in the world. However, vegetation can change flow structure and turbulence characteristics, impacting the use of water energy. In previous researches, few studies have focused on the comparison of velocity and turbulence influenced by various vegetations. Therefore, laboratory experiments were carried out to investigate hydrodynamics affected by submerged rigid vegetation (reed and wooden stick) and merged flexible vegetation (grass and chlorella) under different conditions. The time-averaged velocity distributions of planted floodplain are not logarithmic. Instead, reed and wooden stick followed an "S-shape" profile, but for grass and chlorella, they presented reverse S-shape profile. For all cases, turbulence is not isotropic and the change law of turbulence intensity is different in different sections.
\end{abstract}

\section{Introduction}

The vegetation, as a significant ecological element of natural rivers and riparian systems, plays an important role in the river bio-engineering. It can generally have an important role in altering the flow resistance, the flow structure and distribution, as well as sediment suspension and transport rate and consequently impacting on the ecosystem and morphology greatly [1][2][3]. Therefore, aquatic and riparian vegetation has recently garnered considerable attention in the management and restoration of rivers and coastal ecologies [4] [5].

Effects of vegetation on flow have drawn wide attentions. Although many studies have investigated flow characteristics in vegetated open-channel flows, the flow and turbulence profiles within submerged rigid and merged stiff vegetation remain unclear [6]. Thus, in this paper, the flow velocity and turbulence intensity of various types of vegetations under different hydrodynamic conditions were investigated and compared.

The paper is organized as follows. Sections 2 describes the flume setup and experimental scheme. Section 3 presents the theoretical background. The effects of emerged rigid vegetation and submerged flexible vegetation on velocity and turbulence intensity distribution are discussed in Section4. This paper concludes in section5. The primary objective of this study was to investigate the influence of various vegetation on the hydrodynamic characteristics of vegetated channel.

\section{Materials and methods}

\subsection{Experiment apparatus}

Currently, indoor flume drainage experiments are mostly used to study the flow characteristics of vegetation. In this paper, all the experiments were conducted at the International Research Centre for Coastal and Offshore Engineering of Zhejiang University. The experiment device (Figure 1) includes a rectangular water tank, a pressure gauge, a tailgate and an electromagnetic flow meter. The bottom of tank and both walls were made of Plexiglas. The tank was $47 \mathrm{~m}$ long, $0.6 \mathrm{~m}$ wide, and $0.8 \mathrm{~m}$ deep. The circulation of currents was ensured by the underground circulation system during the experiment.

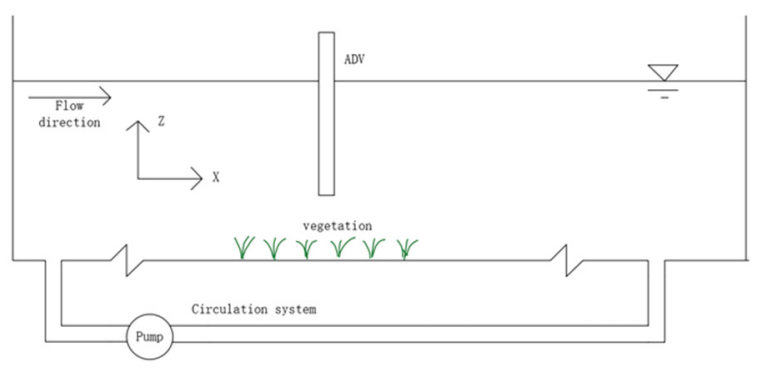

Fig. 1. Experiment setup: schematic of the flume (long view, not to scale)

\footnotetext{
* Corresponding author: 21934107@zju.edu.cn
} 
The Acoustic Doppler Velocimeter (ADV) manufactured by Son Tek Inc., (San Diego, CA, USA) was used to measure the flow characteristics (Figure2).

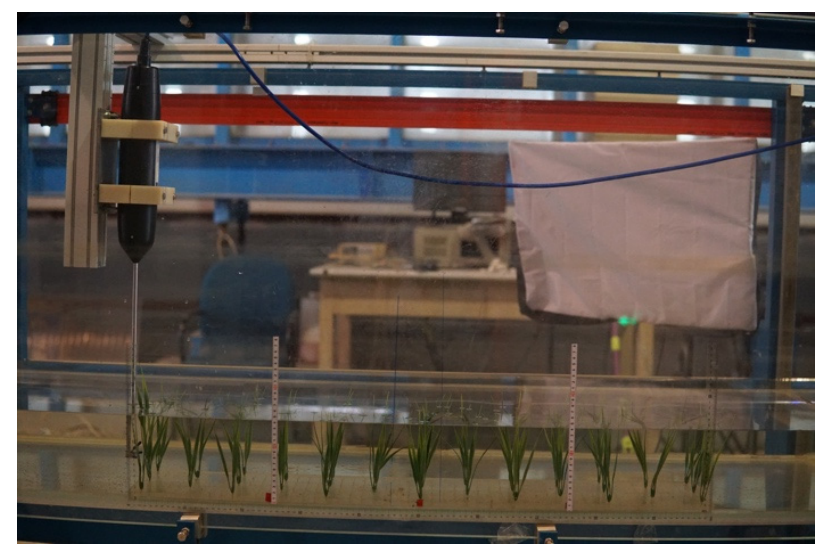

Fig. 2. Layout diagram of ADV

It was mounted in a metal frame of each test area. In addition, it could be easily moved upstream and downstream ensuring that all the measurements were vertically aligned. The components of velocity, $u, v$ and $w$ correspond to the streamwise $(x)$, lateral $(y)$, and vertical $(z)$ directions, respectively. During the current propagation, the ADV was utilized at a sampling frequency of $25 \mathrm{~Hz}$ with 60 s sampling time[6]. Thus, 1500 data were acquired to estimate the average value for each measurement point. In order to ensure the repeatability and accuracy of the experiment, all tests were carried out at least twice.

\subsection{Vegetation materials}

To present four different types of vegetations on the floodplain, the researchers chose reed and wooden stick as rigid submerged plants, plastic grass and chlorella as flexible emerged plants, respectively. The experimenter placed the mimic vegetation by putting various plants in the prepared holes drilled in the PVC board with dimensions of $1 \mathrm{~m} \times 0.1 \mathrm{~m} \times 0.005 \mathrm{~m}$ (length, width and thickness), ignoring swaying and bending. For each type of vegetation, the density of vegetation is $200 \mathrm{stems} / \mathrm{s}^{2}$. In addition, the patterns were the same for each type of vegetation.

\subsection{Experiment conditions}

In this experiment, at the beginning of the test area, a set of three flow depths $h(0.15 \mathrm{~m}, 0.2 \mathrm{~m}, 0.25 \mathrm{~m})$ and five discharges Q $(10,15,20,25,30 \mathrm{~L} / \mathrm{s})$ were adopted. Flow in all rest runs was non-uniform. It is fully recognized that, although the size and scale of the experiment flume and experimental setup are small, albeit with small Reynolds numbers, the data can make sense under turbulent flow conditions. Regardless of scale, the structure of turbulence intensity is similar in nature. In addition, in the past, laboratory experiments were the sole effective way to obtain adequate data on both temporal and spatial scales. Such data obtained in laboratory have often been directly applicable at full scale [7].

\subsection{Location of flow measurement}

For measurement, the authors arranged five verticals for measurement. Five measurement sections are set equidistant on the plant layout belt with a length of 1 meter, which are denoted as V1, V2, V3, V4 and V5 respectively. Therefore, we measure the velocity distributions at five verticals to investigate possible influences of nonuniformity on the longitudinal development of the velocity profile.

Data for four different types of vegetations, with a total 150 test series, have been tested for obtained in this research. A summary of experimental conditions is presented in Table 1. For all the tests, the Reynolds numbers, $R_{e}$, ranged from approximately 9068-33200, which indicates that they are within the range of turbulence flow. In addition, the Froude numbers, $F_{r}$, ranged from $0.0018-0.0754$, meaning that they can be considered subcritical flow.

\section{Theoretical background}

\subsection{Mean velocity}

$$
u_{i}=\frac{1}{T} \int_{0}^{T} u_{i}(t) d t
$$

Where $u_{i}$ is the mean velocity; $T$ is time.

\subsection{Turbulence intensity}

Quantities of analysis was carried out basing on measurements of velocity fluctuations at one point in the flow. In this research, both the mean flow velocity elements $(u, v, w)$ and velocity fluctuation components in turbulence $\left(u^{\prime}, v^{\prime}, w^{\prime}\right)$ correspond to the streamwise, lateral and vertical directions, respectively. Velocity fluctuations can be regarded as the deviation from the mean velocity. In general, root-mean-square (RMS) of velocity fluctuation is considered to be a measure of turbulence intensity. Turbulence intensities corresponding to three different directions: streamwise, lateral and vertical directions are defined as follows:

$$
\begin{aligned}
R M S_{u} & =\sqrt{\left(\Sigma{u^{\prime}}^{2}\right) / N} \\
R M S_{v} & =\sqrt{\left(\Sigma{v^{\prime}}^{2}\right) / N} \\
R M S_{w} & =\sqrt{\left(\sum{w^{\prime}}^{2}\right) / N}
\end{aligned}
$$

Where $N$ is the total number of observations in a given sequence; $u^{\prime}, v^{\prime}$, and $w^{\prime}$ are velocity fluctuations of streamwise, lateral and vertical flow, respectively; $R M S_{u}, R M S_{v}$ and $R M S_{w}$ are turbulence intensities corresponding to streamwise, lateral and vertical flow, respectively.[6]

\section{Results and discussions}

\subsection{Mean velocity profile}

Velocities at one point can be measured in three mutually 
perpendicular directions: streamwise ( $u$,parallel to the boundary,), lateral ( $v$,normal to the boundary), and vertical $(w)$. Thus, researchers use the several test runs to investigate the flow velocities and turbulence intensity, respectively, in $\mathrm{x}, \mathrm{y}$, and $\mathrm{z}$ directions. Figure 5 presents the measured velocity patterns on the plane $z / h$ against $u / U$, where $z$ is the vertical length from the bed, $h$ is the water depth and $U$ means the shear velocity, calculated by the equation $U=\sqrt{g h J}$, in which $J$ is the energy slope.

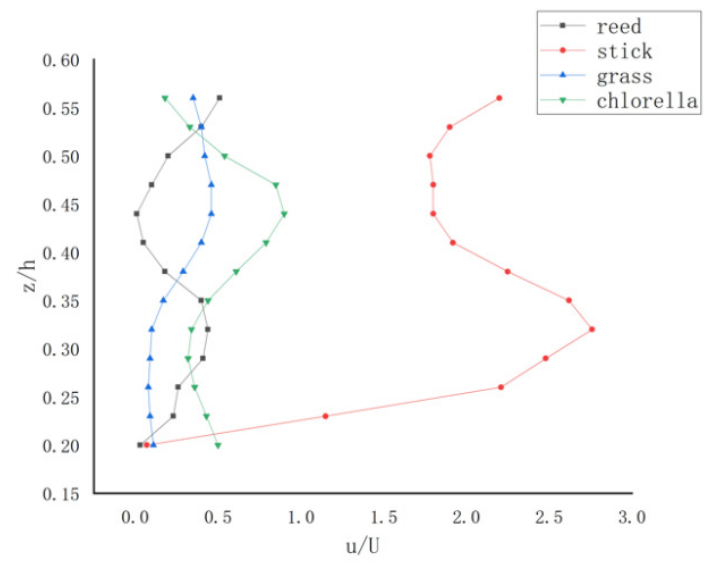

Fig. 3. Vertical distributions of point velocity for different types of vegetation of $Q=20 \mathrm{~L} / \mathrm{s}$, at the vertical 4

Figure 3 represents the vertical distribution of the timeaveraged velocities at the vertical 4 when the Q is $20 \mathrm{~L} / \mathrm{s}$. It represents that the mean flow after vegetation planted is obviously slowed and does not follow logarithmic distribution anymore.

Obviously is different among four different vegetations. Firstly, as submerged rigid vegetation, both reed and wooden stick follows "S" shape. However, as merged flexible vegetations, grass and chlorella follow opposite "S" shape. Therefore, the shape of profile may lie on the type of the vegetation. Figure 3 also presents that the relationship between the height of plant and the water depth influences on the distribution of vertical velocity. Furthermore, they vary significantly in mean velocity distributions and exhibit the formation of a horizonal shear layer. The magnitude of the mean velocity for the wooden stick is more than others because the wooden stick consumes much less energy and momentum from the flow through the generation of turbulence. This result is similar to the paper by[7]. In Yang 2007's study, three zones are divided by the "S-shape" pattern and the degree of each region is related to flow depth and vegetation type[7]. For the rigid vegetation river course, the resistance of plants after planting reduces the mean velocity of water flow, and the velocity of section is redistributed. The velocity of plant area does not obey the logarithmic distribution law any more, and the velocity distribution of each section is different. Current velocity stratification is not obvious. For the flexible vegetation channel, the vegetation fluctuation and deflection phenomenon make the water flow becomes unstable and the vertical flow velocity decreases. The vegetation curve stream line and fluctuate obviously, and the top of vegetation layer has a tendency to be flattened by water. The velocity distribution near the top area of the vegetation layer presents a semilogarithmic distribution. The vertical velocity of water flowing through the vegetation area shows an S-shaped distribution, which is mainly due to the structure of the vegetation itself.

\subsection{Turbulence intensity}

The transportation and settlement of sediment in the river course, the erosion of riverbed embankment and the resistance of flow are all affected by the flow turbulence. With the increasing maturity and perfection of threedimensional turbulence numerical simulation, it is very important to verify these models with high quality test data. Therefore, it is significant to study the flow turbulence intensity.

Figure 4-6 shows the distribution of turbulence intensities, $R M S_{u}, R M S_{v}, R M S_{w}$ against the relative depth $z / h$ at the Vertical5 (V5) of different vegetations with $\mathrm{Q}=30 \mathrm{~L} / \mathrm{s}$. These changes correspond to the vertical distribution of velocity along the flow points. In the case of vegetative floodplain, the vertical distribution of turbulence intensity along the flow and transverse turbulence intensity is also S-shaped. However, the type of vertical turbulence intensity distributions in each direction are not the same kind. The phenomenon was found that the streamwise and lateral intensities are approximately equal by comparing the distribution in three different directions.

According to the figure 4-6 shows that the water near the surface part, three directions of longitudinal, lateral and vertical turbulence intensity is bigger, it may be that the interaction of water and plants to produce turbulent vortex and interface wave, that has a strong quality and momentum exchange here, and somewhere in the region reached a maximum, and near the surface are decreased. Figure 4-6 also shows that the turbulence intensity distribution is anisotropic.

It can also be analysed from the figure that the vertical turbulence intensity and turbulence intensity distribution law of vertical and horizontal difference is bigger, and vertical turbulence intensity is less than the streamwise and lateral, which is due to the turbulent vortex in the statistical measure of vertical is less than the horizontal scale of turbulence did not reach isotropic. This is due to water flow interactions with plants and walls that limit the scale of vertical turbulence.

For the river course with rigid vegetation, the turbulence intensity in the three directions near the water surface is larger and reaches the maximum in this area, while it decreases at the area near the water surface. Turbulence is not isotropic. The change law of turbulence intensity is different in different sections. For the channel with flexible vegetation, strong mass and momentum exchange exists at the top of the planted area, and the turbulence intensity is relatively large in the vertical, horizontal and vertical directions. Because the statistical scale of the turbulent vortex in the vertical direction is obviously smaller than that in the horizontal direction, the turbulence does not reach isotropy. The change law of turbulence intensity is also different in different sections. 
This is consistent with the distribution law of turbulence intensity in the river channel with rigid vegetation.

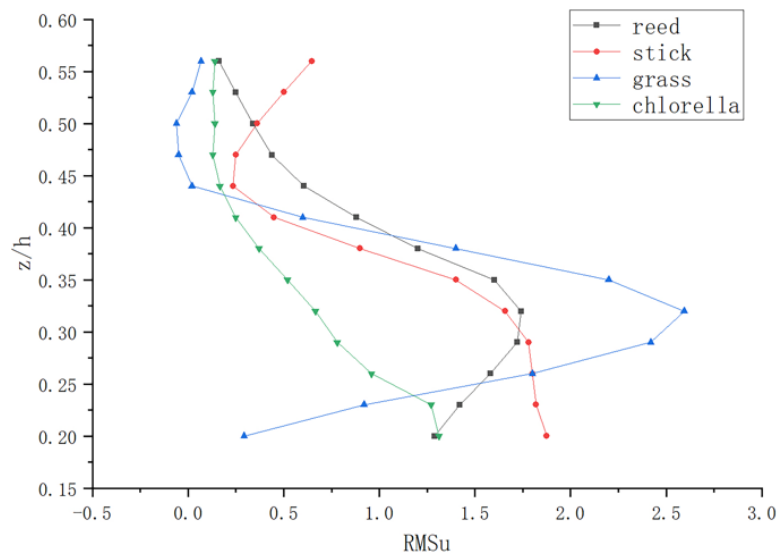

Fig. 4. Turbulence intensities $R M S u$ against relative depth ratio of vertical distance from bed $\mathrm{z}$ to depth $\mathrm{h}$, represents $\mathrm{V} 5$ and $\mathrm{Q}=30 \mathrm{~L} / \mathrm{s}$

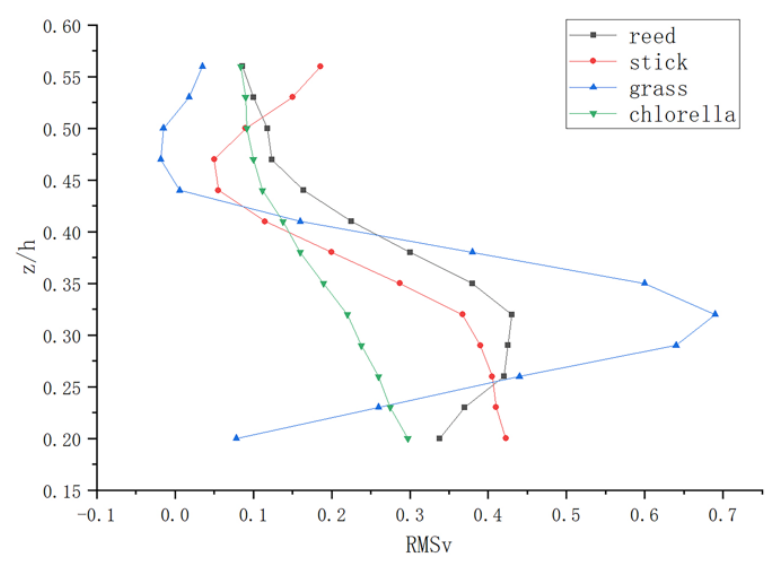

Fig. 5. Turbulence intensities $R M S v$ against relative depth ratio of vertical distance from bed $\mathrm{z}$ to depth $\mathrm{h}$, represents $\mathrm{V} 5$ and $\mathrm{Q}=30 \mathrm{~L} / \mathrm{s}$

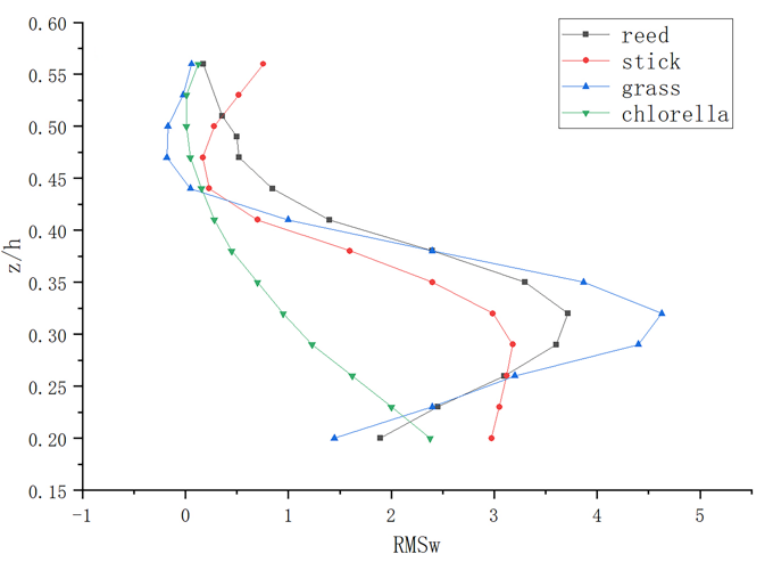

Fig. 6. Turbulence intensities $R M S w$ against relative depth ratio of vertical distance from bed $\mathrm{z}$ to depth $\mathrm{h}$, represents $\mathrm{V} 5$ and $\mathrm{Q}=30 \mathrm{~L} / \mathrm{s}$

\section{Conclusions}

These small-scale experiments reported that the shape of profile may lie on the type of the vegetation. The distribution of streamwise mean velocity for emerged rigid vegetation follows an S-shape curve that exhibits three regions, different for the various types of vegetation, as shown in Figure 3. As for merged flexible vegetations, grass and chlorella follow opposite "S" shape.

In addition, the vertical distributions of streamwise and lateral turbulence intensities are S-shaped for vegetated floodplains, similar to the distributions of streamwise point velocity. The turbulence intensity, however, does not follow the same kind of distribution. The streamwise and lateral turbulence intensities are approximately equal. The vertical turbulence intensity is the weakest.

Based on the experimental work, a better understanding of flow characteristics due to different types of natural stiff and flexible vegetation under merged and submerged vegetation can be derived from the data. In addition, the data can be applied into numerical models. Furthermore, the experiments on natural plants also provide a powerful reference basis for other investigations utilizing artificial vegetation.

\section{References}

1. Ackerman, J.D.; Okubo, A. Reduced Mixing in a Marine Macrophyte Canopy. Funct. Ecol. 1993, 7, 305, doi:10.2307/2390209.

2. Nepf, H.M.; Vivoni, E.R. Flow structure in depthlimited, vegetated flow. J. Geophys. Res. Ocean. 2000, 105, 28547-28557, doi:10.1029/2000jc900145.

3. Luhar, M.; Rominger, J.; Nepf, H. Interaction between flow, transport and vegetation spatial structure. Environ. Fluid Mech. 2008, 8, 423-439, doi:10.1007/s10652-008-9080-9.

4. Leonard, L.A.; Luther, M.E. Flow hydrodynamics in tidal marsh canopies. Limnol. Oceanogr. 1995, 40, 1474-1484, doi:10.4319/1o.1995.40.8.1474.

5. Kemp, J.L.; Harper, D.M.; Crosa, G.A. The habitatscale ecohydraulics of rivers. Ecol. Eng. 2000, 16, 17-29, doi:10.1016/S0925-8574(00)00073-2.

6. Chen, S.C.; Kuo, Y.M.; Li, Y.H. Flow characteristics within different configurations of submerged flexible vegetation. J. Hydrol. 2011, 398, 124-134, doi:10.1016/j.jhydrol.2010.12.018.

7. Yang, K.; Cao, S.; Knight, D.W. Flow Patterns in Compound Channels with Vegetated Floodplains. $J$. Hydraul. Eng. 2007, 133, 148-159, doi:10.1061/(asce)0733-9429(2007)133:2(148). 